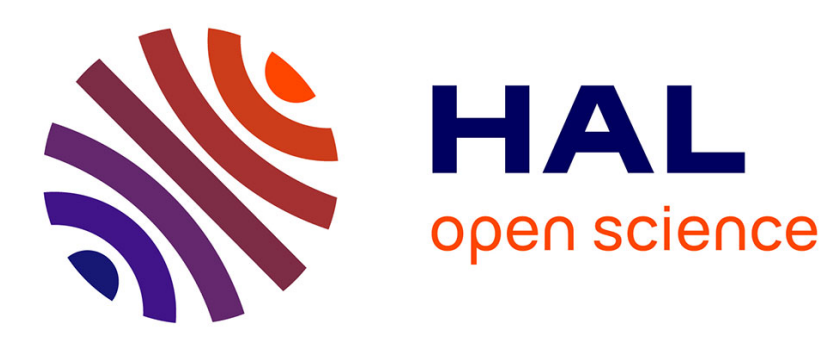

\title{
Non-Homologous End-Joining at challenged replication forks: an RNA connection?
}

\author{
Charlotte Audoynaud, Stephan Vagner, Sarah Lambert
}

\section{To cite this version:}

Charlotte Audoynaud, Stephan Vagner, Sarah Lambert. Non-Homologous End-Joining at challenged replication forks: an RNA connection?. Trends in Genetics, 2021. hal-03358678

\section{HAL Id: hal-03358678 \\ https://hal.science/hal-03358678}

Submitted on 29 Sep 2021

HAL is a multi-disciplinary open access archive for the deposit and dissemination of scientific research documents, whether they are published or not. The documents may come from teaching and research institutions in France or abroad, or from public or private research centers.
L'archive ouverte pluridisciplinaire HAL, est destinée au dépôt et à la diffusion de documents scientifiques de niveau recherche, publiés ou non, émanant des établissements d'enseignement et de recherche français ou étrangers, des laboratoires publics ou privés. 
Non-Homologous End-Joining at challenged replication forks: an RNA connection?

Charlotte Audoynaud ${ }^{1,2,3}$, Stephan Vagner ${ }^{1,2,3}$ and Sarah Lambert ${ }^{1,2,3}$

1 Institut Curie, Université PSL, CNRS UMR3348, INSERM U1278, 91400 Orsay, France

2 Université Paris-Saclay, CNRS UMR3348, INSERM U1278, 91400 Orsay, France 3 Equipes Labélisées Ligue Nationale Contre Le Cancer, 91400 Orsay, France

corresponding author: sarah.lambert@curie.fr

Keywords: DNA replication fork, single- and double-ended Double-Strand Break, Non-Homologous End-Joining, RNA, Genome Stability 


\begin{abstract}
Defective DNA replication, known as replication stress, is a source of DNA damage, a hallmark of numerous human diseases including cancer, developmental defect, neurological disorders and premature aging. Recent works indicate that NonHomologous End-Joining (NHEJ) is unexpectedly active during DNA replication to repair replication-born DNA lesions and to safeguard replication fork integrity. However, erroneous NHEJ events are deleterious to genome stability. RNAs are novel regulators of NHEJ activity through their ability to modulate the assembly of repair complexes in trans. At DNA damage sites, RNAs and DNA-embedded ribonucleotides modulate repair efficiency and fidelity. We discuss here how RNAs and associated proteins including RNA binding proteins (RBPs) may regulate NHEJ to sustain genome stability during DNA replication.
\end{abstract}




\section{Double-ended Double Strand Break Repair Pathways}

Double-ended DNA Double-Strand Breaks (deDSBs) are considered as the most toxic lesions since they are drivers of chromosomal rearrangement. These deDSBs are repaired by two distinct pathways: Homologous Recombination (HR) and NonHomologous End-Joining (NHEJ) (See Glossary) [1]. HR requires the presence of an intact homologous sequence and is initiated by a well-orchestrated nucleolytic degradation (named end-resection) of DSB ends to expose single-stranded DNA (ssDNA) [2]. The canonic NHEJ (cNHEJ) pathway acts at deDSBs by rejoining the two DNA extremities with none or little end trimming, regardless of sequence homology [3]. cNHEJ factors, such as $\mathrm{KU}, 53 \mathrm{BP} 1$ and RIF1, counteract the resection step, which is key for deDSB repair pathway choice [4]. When a limited end-resection is engaged, an error-prone Alternative End-Joining (Alt-EJ) can exploit micro-homologies to anneal and ligate DNA ends [3].

Replication-associated DNA damages usually rely on HR for error-free repair. Nevertheless, several reports indicate that NHEJ is also active during DNA replication in unicellular organisms and in metazoan, supporting evolutionarily conserved functions in sustaining cell proliferation upon Replication Stress (RS). Many NHEJ factors are engaged at damaged replication forks, althought not in their canonical functional mode to drive DNA transactions, either beneficial or toxic for genome stability. Here, we discuss the distinct functions of NHEJ in DNA replication and how RNAs may contribute to regulate its activity to avoid mutagenic repair of replicative DNA lesions.

\section{End-joining factors are active during DNA replication}

RS is an underlying source of genome instability [5]. Converging results indicate a role for NHEJ factors in safeguarding the integrity of newly replicated strands, in repairing replication-induced DSBs and in restarting replication forks (Figure 1). In many organisms, the lack of NHEJ factors leads to cellular sensitivity to various replicationblocking agents $[6,7]$ and mammalian core NHEJ components (KU, DNA-PKcs, 53BP1, RIF1) are found at newly replicated DNA of halted forks [8-10], althought their precise functions are not always understood. When the major components of $\mathrm{HR}$ are lost, the repair of replication-associated DSBs relies on Alt-EJ factors such as PolQ, but NHEJ factors can also cooperate with HR to repair replicative DNA damage [11,12].

Upon head-on collisions between the transcription and replication machineries, the nascent RNA has the tendency to anneal with its DNA template to form a R-loop, contributing to fork stalling (Figure 1A) [13]. Enzymatic cleavage of the stalled fork is proposed to facilitate RNA polymerase II (RNAPII) progression. The cleaved fork is then repaired by the co-action of Rad52 and the Ligase4-Xrcc4 complex, known to join DNA ends during DSBs repair by NHEJ, allowing replication restart [13]. This type of EJ-dependent fork repair likely ensures, together with DNA-PKcs, proper cell proliferation and resistance to RS in human triple negative breast cancer [14]. 
When active replication forks encounter a DNA nick, it results in the formation of a broken fork from which one sister chromatid is teared off. This replicative DNA lesion called single-ended DSB (seDSB) can be further converted into a deDSB when the converging fork reaches the seDSB site (Figure 1B) [15]. The topoisomerase I inhibitor campthotecin (CPT), which derivatives are used in chemotherapy, considerably increased the occurrence of seDSBs and KU is an early responder to CPT-induced broken forks [16,17]. Even if NHEJ at seDSBs is a dead-end, in terms of ligation of DNA extremities, numerous studies in yeast have revealed that KU binds seDSBs and restricts HR by blocking long-range end-resection, unless it is evicted by the coordinated action of endonucleases such as MRN (Mre11-Rad50-NBS1) [18-21]. Mammalian KU also engages at CPT-induced stalled forks and is evicted by the coordinated action of MRN and CtIP (Figure 1B). Surprisingly, the persistence of KU at seDSBs rather limits RAD51 loading than the long-range resection, suggesting that $\mathrm{KU}$ binding to seDSBs could be a first step of the HR process to repair broken forks [22].

In a process named replication fork protection, nascent DNA is protected from nuclease attacks, a step necessary to stabilize halted fork and likely to favor its merging with a converging fork [23]. Upon various types of RS, active forks are converted into reversed forks, four-way DNA structures in which the newly replicated strands are annealed together, exposing a double-stranded DNA end (dsDNA end), distinct from a seDSB (Figure 1C) [24]. Although the controlled degradation of nascent strands is necessary to expose ssDNA to recruit HR factors [2,23], an excessive degradation, occurring when the RAD51 filament is not formed appropriately, is toxic to cell survival and contributes to breast cancer sensitivity to chemotherapies [24]. In both yeast and mammals, some NHEJ components, such as KU, XLF, RIF1 and 53BP1, act at reversed forks as anti-resection barriers, independently of their canonical function in NHEJ, to stabilize halted forks and facilitate replication restart (Figure1C) $[9,19,25-$ 28]. For instance, fission yeast KU binds arrested forks to orchestrate the resection of newly replicated strands, independently of ligase 4, and to allow timely replication restart, albeit the molecular mechanism of this last function remains unclear [19]. Besides, in mouse embryonic fibroblastes, RIF1-mediated protection of stalled forks requires its interaction with the Protein Phosphatase 1 but is independent of its interacting partner 53BP1 $[9,27]$. However, in cancer cells, 53BP1 was found necessary for protecting stalled forks from degradation [26]. NHEJ components therefore constitute essential factors for replication fork protection and stabilization, contributing to maintain genome stability upon RS, likely in a cell-type dependent manner.

While NHEJ factors promote replication restart and fork-integrity, their activity should be regulated to avoid toxic repair events. When replication termination occurs at a broken fork, the seDSB is converted into a deDSB in one sister chromatid, which ligation by NHEJ may be beneficial to complete DNA replication (Figure 1B). However, ligation of distant seDSBs would generate chromosomal rearrangements [15]. ATM, a maestro kinase of the DNA Damage Response (DDR) counteracts toxic Ligase4- 
mediated repair of seDSBs, events that underlie the high sensitivity of ATM-deficient cells to CPT derivatives [29]. It is proposed that ATM activity at seDSBs allows KU and DNA-PKcs removal to prevent aberrant ligation events promoting instead HR-mediated repair (Figure 1B) [30]. In addition, in the absence of BRCA1, NHEJ has been also involved in the formation of micro-homology-mediated tandem duplications occurring at a site-specific replication fork barrier, a type of rearrangement akin to a mutational signature observed in ovarian cancers lacking BRCA1 [31]. While NHEJ is cell cycleregulated with CYREN (cell cycle regulator of NHEJ), preventing KU binding to break sites with overhangs in S and G2 phase [32], little is known about the regulation of NHEJ activity once core components are engaged at seDSBs or reversed forks. Such knowledge is necessary to improve cancer treatment and overcome chemo-resistance. The emerging role of non-coding RNAs (ncRNAs) in fostering the DDR and the fact that several NHEJ components are identified in large-scale proteomic studies as putative RNA Binding Proteins (RBPs) point towards a role for RNA in regulating NHEJ activity.

\section{RNAs as regulators of genome stability at DNA damage sites}

RNAs have emerged as having opposite roles in genome maintenance and in promoting genome instability [33]. RNAs act through several means (Figure 2): i) de novo transcribed-RNAs at DNA damage sites foster DDR, ii) RNAs act as scaffolds for the association of DDR factors to modulate repair activities, iii) RNAs associated to the DNA template interfere with DNA repair processes but also lead to DNA injuries. We will discuss how those 3 RNA facets could contribute to modulate NHEJ activities at RS sites.

\section{RNAs at Double-Strand Break sites}

Recent works have revealed that deDSBs in mammalian cells can behave as RNAPII promoters to generate Damage-induced long ncRNAs (referred to as dilncRNAs) from both sides of deDSBs (text box) [34-37]. The MRN complex triggers RNAPII recruitment and activity at deDSBs, before engagement towards the step of endresection $[34,38]$. DilncRNAs can then contrbite to RNA:DNA hybrids and double stranded RNAs (dsRNAs) formation, the latest being processed into shorter DNA Damage Response RNAs (DDRNAs). DDRNAs, together with dilncRNAs, contribute to the focal assembly of deDSBs signaling and repair factors such as the NHEJ factor 53BP1 (Figure 2A), a topic that has been recently reviewed in depth $[33,35,38]$. Interestingly, RNA:DNA hybrids have been detected at deDSBs in both yeast and human cells and several mechanisms underly their formation (Figure $2 B$ and $C$ ) [36,39-45]. First, nascent dilncRNAs may anneal to the resected DNA ends (Figure 2B). Second, following RNAPII stalling favored by deDSBs induction, pre-existing nascent RNAs may be liable to anneal with the DNA template, akin to cotranscriptionnal R-loop formation (Figure 2C). One unanswered question in the field is to determine whether the RNA moiety of RNA:DNA hybrids correspond to pre-existing nascent RNA or to de novo transcription at deDSBs sites, thought a recent study in human supports the last hypothesis [44]. 
Moreovre, it is currently unclear to which extent RNA:DNA hybrids are obligatory intermediates during deDSB repair or are rather opportunistic by-products of RNAPII activity, a point discussed in depth in [46]. In human cells, RNA:DNA hybrids have been proposed to help recruiting HR factors such as BRCA1 and BRCA2 (text box) [43]. Akin to findings in fission yeast, RNA:DNA hybrids were proposed to limit ssDNA exposure and to protect ssDNA 3'-overhang from degradation in human cells $[39,44]$. Despite acting positively on DNA repair, there is a need of RNA:DNA hybrids clearance at deDSBs. Indeed, long RNA:DNA hybrids inhibit the long-range resection mediated by Exo1 in vitro [47]. Consistent with this, several studies reported that RNA:DNA hybrids dismantling activities are at work at deDSB sites, including human Senataxin, RNaseH2, the exosome, and the DDX1 and DDX5 RNA helicase [40,43,48-50]. An emerging view from the literature is that persistent RNA:DNA hybrids at deDSBs constitue an impediment to DSB repair making deDSBs more prone to engage in translocation events [40]. However, recent works have challenged this view suggesting that depending on the context, RNA:DNA hybrids, broadly RNA species, at deDSBs influence repair pathway choice as further discussed.

\section{RNAs during DNA replication}

Many ribonucleotides are present in the DNA template during replication (Key Figure

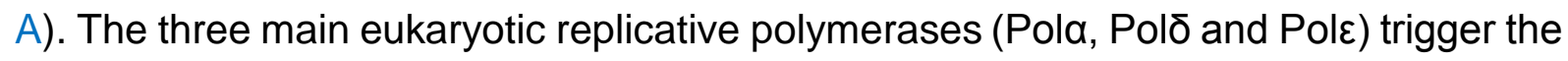
misincorporation of rNMPs into newly synthesized DNA at an average of one ribonucleotide per $1.5-2 \mathrm{~Kb}$ in human cells [33]. DNA-embedded ribonucleotides provide a signal of strand specificity to guide mismatch repair [51]. When not removed by $\mathrm{RNase} \mathrm{H}$ activities, stretches of ribonucleotides impair fork progression and the resolution of topological constraint by Topoisomerase I [33]. Besides, on the lagging strand template, the Pol a-primase synthesizes short RNA:DNA primers that are subsequently extended by Polठ to form Okazaki fragments (OFs) [52]. Though quickly maturated after fork passage, primase-mediated RNA primers occur every 100-300 bp and thus constitute an unavoidable source of RNA:DNA hybrids during DNA replication Their potential influence on fork-repair, as decribed for deDSBs repair, is unknown (Key Figure A).

Whether a focal RNA-mediated DDR occurs at RS sites remains poorly documented (Key Figure B). Because both dsDNA ends formed at a reversed fork and seDSB engage classical DDR factors such as KU, MRN and 53BP1 to foster ATM signaling [53], it raises the possibility that a focal DDRNA response may take place at RS sites, as described for deDSBs. In support of this, replication-blocking agents and difficultto-replicate genomic elements lead to the production of a particular class of ncRNAs, called qiRNA in Neurospora crassa [54,55]. The biogenesis of qiRNA requires the quelling pathway, an RNAi-related mechanism, as well as, active DNA replication, the ssDNA binding protein RPA and HR factors. Interestingly, qiRNA helps to avoid end-joining events within repeated sequences leading to genome instability, but the precise underlying mechanism remains unknown. 
Physiological R-loops form dynamically at transcription promoters, terminators and in gene bodies to regulate gene expression [56,57]. Unresolved R-loops worsen transcription-replication conflicts leading to fork stalling and, when enzymatically cleaved, to seDSBs. In human cells, nascent chromatin is transcriptionally silent and inaccessible to RNA polymerases. Transcription restart, that contributes to the maturation of post-replicative chromatin, is timely separated from the DNA replication process [58]. However, several RNA helicases, such as the yeast Sen1, and human RNA helicases such as DDX1, DDX5, DDX21 and DHX9, associate with replication forks to unwind potential R-loops and, upon RS, to avoid their formation de novo behind stalled forks, especially when the replication factor PCNA is not timely unloaded from nascent DNA [59-61]. It indicates that, in some circumstances, RNAPII can engage shortly after fork passage. A better understanding of the coordination of the transcription activity with fork progression and processing is necessary to appreciate how RNAs present at RS sites contribute to genome maintenance and maybe to fork repair pathway choice (Key Figure).

\section{RNAs regulate NHEJ activities}

Recently, large-scale proteomics studies identified numerous DDR and NHEJ proteins as putative RBPs, albeit most of them do not carry classically characterized RNA binding domains (Table 1) [62-68]. We detail below how the potential association of these proteins with RNAs could modulate NHEJ activity, especially in a RS context.

Recent studies showed that 53BP1 foci form in a transcription and RNA-dependent manner at deDSB sites (Figure 2 A) $[35,38,69]$. RNA immunoprecipitation assays have shown that 53BP1 associates with RNAs and DDRNAs [36,70]. Large-scale proteomics studies also identified 53BP1 as a candidate protein directly interacting with RNA $[66,67]$. Of note, 53BP1 as well as MRE11, contain a GAR domain, known to be responsible for direct RNA binding in other proteins [71-73]. It is possible that RNAs transcribed at deDSBs help to recruit 53BP1 and other NHEJ proteins, while fostering its intrinsic ability to promote phase-separation, a step necessary to recruit downstream DNA repair factors [38]. However, direct evidences that the ability of 53BP1 to bind RNAs is a key property to ensure 53BP1-dependent DDR signaling are still missing. In addition, a characterized RBP (called TIRR) interacts with 53BP1 and masks its Tudor domain, that itself binds methylated histones and the dimethylated form of the cell-cycle regulator p53 [74,75]. It cannot be excluded that DSB-induced RNAs production at deDSBs act in trans to displace TIRR-53BP1 interaction making 53BP1 available for recruitment to deDSBs in a histone-dependent manner. Additionnal knowledge is needed to establish how the potential RBP feature of 53BP1, as well as others DDR factors contribute, to DNA Damage signaling and repair. Interestingly, the recruitment of 53BP1 to deDSBs is increased following the silencing of the two estrogen-regulated CUPID1 and CUPID2 long ncRNAs concomitant with a reduced deDSB end-resection in human breast cancer cell lines (Figure 2D) [76]. Although the underlying mechanism is still unknown, it illustrates the possibility that ncRNAs may modulate the anti-resection function of 53BP1, a potential appealing 
mechanism of fork protection by 53BP1. Altogether, this raises the question about the extent to which 53BP1 functions, both at deDSBs and at RS sites, are modulated by its association with RNAs.

Direct RNA binding is a conserved function of KU that tends to bind to structured RNA stem-loops with bulged-out nucleotides and RNA G-quadruplexes [77-79]. KU interacts with a number of different RNAs including the telomerase RNA TLC1 for telomere end maintenance, mRNAs and ncRNAs such as LINP1 (IncRNA in NHEJ pathway 1) [80-83]. KU binds to several structured RNA motifs in LINP1, which itself is capable of forming phase-separated condensates through RNA-RNA interaction, to promote KU multimerization and stabilization of the initial synaptic event for NHEJ (Figure 2E) [79]. LINP1 also acts as a scaffold that stabilizes the interaction between KU80 and DNA-PKcs, thereby promoting NHEJ and radiation resistance in human triple negative breast cancer cells, where LINP1 is overexpressed [82]. Whether LINP1 is involved in modulating NHEJ activity during S-phase is currently unknown. Importantly, KU binding to the telomerase RNA can stimulate the kinase activity of DNA-PKcs that therefore mediate protein phosphorylation in an RNA-dependent manner [84]..Although less molecularly documented, the IncRNA LRIK, the expression of which increases upon induction of DSBs, associates with KU to enhance the recruitement of cNHEJ factors to damaged chromatin and repair activity [85]. Such discoveries highlight the potential of scaffolding RNAs in the regulation of NHEJ activity.

RNA-templated DNA repair is an emerging mode of repair in the field of DSBs repair, yet the underlying mechanisms are only beginning to be elucidated [33]. Pioneering works in yeast have revealed how the HR process used RNA template for homologybased repair, a potentially useful mechanism when the intact sister chromatid is not available for repair [86]. Thus, it is worth considering that RNAs biogenesis at deDSBs occurs when all DNA templates are cutted by enginerred nucleases employed to target DSBs at specific loci, making DNA-templated DSB repair unavailable. In human, deDSBs at actively transcribed chromatin are preferentially repaired by $\mathrm{HR}$, but recent works showed that RNA transcripts can also guide deDSBs repair by NHEJ (Figure 2 C) $[33,87]$. Mammalian NHEJ factors (such as Ligase4, 53BP1, KU, DNA-PKcs, PNKP, XRCC4) interact with the elongating RNAPII and/or proteins involved in RNA processing such as SETX and XRN2 [88-90]. In human embryonic cells, the nascent RNA, preexisting to deDSBs induction, may anneal to the broken DNA matrix to form RNA:DNA hybrid, acting as an anchor to stabilize DNA ends and facilitate NHEJ repair and/or potentially serving as a template for an RNA-dependent DNA polymerase [90]. Additionnaly, KU and the intrinsically disordered RNA-binding protein RBM14 were proposed to trigger RNAPII-dependent RNA:DNA hybrids formation at deDSBs, underscoring the profound and complex relationship between NHEJ and RNAs [90]. Interestingly, human KU binds to RNA:DNA hybrids, though with twice less affinity than to dsDNA [91]. In yeast, the persistence of RNA:DNA hybrid structures at deDSBs favors KU binding and may engage deDSB repair toward NHEJ instead of HR [45]. Nonetheless, opposite conclusions were drawn in human cells in which unresolved 
RNA:DNA hybrids would act as a barrier against cNHEJ [88]. Further investigations are needed to clarify the role of RNA:DNA hybrids in DSB repair pathway choice, likely modulated by the way they form, their associated epigenetic modifications and the chromatin environement $[92,93]$.

RNA-templated DSB repair by NHEJ raises questions about its fidelity. End-joining related polymerases can accommodate ribonucleotides incorporation at repair junctions to favor the ligation step during NHEJ [94]. Plasmid-based assays showed that association of cNHEJ factors with mRNAs favor error-free repair but investigating the fidelity of NHEJ repair at chromosomal breacks in vivo remains challenging [90]. Indeed, at nuclease-induced deDSBs, error-free repair by NHEJ would restore the intact DNA sequence liable to successive rounds of cleavage-repair, as shown in yeast [95]. Thus, if RNA-templated NHEJ repair favors an error-free repair mode, this could mask the potential action of NHEJ at transcribed genes.

Whether RNA:DNA hybrids at RS sites modulate the recruitment of NHEJ factors and their activities is an open question (Key Figure). Interestingly, two pathways of replication fork protection were recently revealed in human cells, that may reflect the presence of distinct nucleic acids substrates at reversed forks [26]. RNA-templated fork-repair is so far poorly documented but in vitro studies indicate that nascent RNAs at stalled RNAP can be used to intitate DNA synthesis [96]. Recently, the human and yeast Replication Protein A (RPA), a well-caracterized ssDNA binding protein, was found to bind RNA and to drive RNA:DNA hybrids formation in vitro from which DNA synthesis can be primed [97].

\section{Concluding Remarks}

NHEJ acts in several ways at halted forks to sustain replication dynamics, contributing to the survival of cancer cells in presence of replication-blocking agents. However, the use of NHEJ during replication is at risk of increased chromosomal rearrangements. Largely speaking, the proximity of NHEJ factors to nascent RNAs or RNA:DNA hybrids and their potential to associate with RNA-containing molecules, are likely to represent an underappreciated level of regulation, yet crucial, in fine-tuning the NHEJ pathway. Little is known about the atlas of RS-induced ncRNAs and defining the role of RNAs at RS sites will be of major importance. Recently, ncRNAs were found enriched at unperturbed replication forks. Many RNAs associate in trans, meaning that their synthesis is uncoupled from DNA replication but repeat-containing ncRNAs associate in cis, suggesting that they are transcribed shortly after fork passage [98]. Those RNAs remain associated to chromatin across the cell cycle, suggesting a role in chromatin maturation. Identifying the repertoire of RNAs associated in cis or in trans with halted replication forks, and elucidating their functions will provide key knowledge to appreciate how RNAs modulate replication fork repair and NHEJ activity to maintain genome stability (Outstanding Question box). RNA-templated fork repair need to be investigated as well as the contribution of DNA-embeded RNA species on fork repair pathway choice. RNAs at RS sites may help to create a signaling hub to integrate a 
"decision point" towards NHEJ activity. More generally speaking, there is a need of investigating whether NHEJ and DDR factors directly interact with RNAs and if such binding is important for their repair and DNA damage signaling functions. Such research paths could pave for the identification of novel anti-cancer targets. 
Table 1. RNA-associated end-joining factors and DSBs sensors identified in largescale proteomic studies

\begin{tabular}{|c|c|c|c|c|}
\hline & Complex & Protein & Gene & References \\
\hline \multirow[t]{4}{*}{ cNHEJ } & - & ARTEMIS & DCLRE1C & [54] \\
\hline & \multirow[t]{3}{*}{ DNA-PK } & DNA-PKcs & PRKDC & [54-59] \\
\hline & & KU80 & XRCC5 & [54-60] \\
\hline & & KU70 & XRCC6 & [54-60] \\
\hline \multirow[t]{3}{*}{ DSBs sensor } & \multirow[t]{2}{*}{ MRN } & MRE11 & MRE11-A & {$[58]$} \\
\hline & & RAD50 & RAD50 & [58] \\
\hline & - & PARP-1 & PARP-1 & {$[55,57-59]$} \\
\hline \multirow{2}{*}{$\begin{array}{r}\text { cNHEJ } \\
\text { substitutes }\end{array}$} & \multirow[t]{2}{*}{ SFPQ-NONO } & SFPQ & SFPQ & [54-60] \\
\hline & & NONO & NONO & [54-60] \\
\hline \multirow{9}{*}{$\begin{array}{r}\text { NHEJ } \\
\text { modulators }\end{array}$} & - & RBM14 & RBM14 & [54-60] \\
\hline & - & 53BP1 & 53BP1 & [58-59] \\
\hline & - & RIF1 & RIF1 & {$[54,58-59]$} \\
\hline & - & RAP1 & RAP1 & {$[57,59]$} \\
\hline & - & SUMO1 & SUMO1 & {$[58-59]$} \\
\hline & - & MDC1 & MDC1 & {$[54,58-59]$} \\
\hline & \multirow[t]{3}{*}{ Cohesin } & SMC1-A & SMC1-A & [58-59] \\
\hline & & SMC3 & SMC3 & [58-59] \\
\hline & & SA-2 & SA-2 & [59] \\
\hline
\end{tabular}




\section{Figure 1: NHEJ pathways at stressed replication forks}

(A) NHEJ at transcription-replication conflicts. At site of head-on transcriptionreplication encounter, topological stress favors co-transcriptional R-loop formation. Fork stalling is resolved by Mus81-dependent fork cleavage, allowing the release of topological stress and the progression of RNA polymerase. Fork repair is then achieved by ligation of the break by the NHEJ ligation machinery allowing replication restart. (B) NHEJ at broken replication forks. When a fork encounters a nick in the DNA template, it results in a broken fork harboring a single-ended DSB (seDSB) bound by $\mathrm{KU}$ (light purple circles) and DNA-PKcs (dark purple circle) that form the holocomplex DNA-PK, restricting DNA end resection. DNA-PK eviction requires the nuclease activities of the MRN-CtIP complex regulated by the ATM kinase, allowing to form RPAcoated single-stranded DNA to switch fork repair towards the HR pathway (top panel). At this stage, ATM activity is critical to prevent toxic end-joining events destabilizing genome stability. When the converging fork is approaching, the seDSB is converted into a double-ended DSB (deDSB) that is repaired by the NHEJ ligation machinery allowing the completion of DNA replication. (C) NHEJ as replication fork protection factors. Various replication-blocking agents lead to replication fork reversal, providing a double-stranded DNA end. Reversed forks are an entry point for numerous nucleases degrading nascent strands, a process that becomes toxic to cell survival when not restrained. Several NHEJ factors act at replication forks to protect nascent strands from degradation by distinct mechanisms (see text for details).

\section{Figure 2: Regulation of NHEJ by RNAs in cis and in trans.}

(A) The synthesis of ncRNAs at deDSBs fosters the DNA Damage Response (DDR) signaling allowing 53BP1-dependent DDR focus formation by phase separation. (B) RNA:DNA hybrids at deDSBs can positively or negatively modulate the recruitment of NHEJ factors. (C) At deDSBs in transcribed regions, pre-existing transcripts may form RNA:DNA hybrids to stabilize the DNA extremities, thereby potentially promoting an error-free and RNA-templated NHEJ repair pathway. Interactions between the NHEJ and RNAPII machineries may help such RNA-templated NHEJ repair (double dashed arrow). (D) IncRNAs such as CUPID1 and CUPID2 limits the anti-resection function of 53BP1 at deDSBs. (E) IncRNAs such as LINP1 and TLC1 act as scaffolds to stimulate DNA-PK kinase activity towards ligation events. (See text for details).

\section{Key figure: RNAs and NHEJ at replication forks}

(A) RNAs species co-existing with the DNA replication process: co-transcriptional Rloops, ribonucleomonophosphates (rNMPs) incorporated by DNA polymerases, and RNA primers of Okazaki fragments forming RNA:DNA hybrids As proposed at deDSBs, those RNA species may play a role in modulating NHEJ activity as well as promoting RNA-templated fork-repair. (B) Replication stress induces single-ended DSBs (seDSBs) that can be further converted into double-ended DSBs (deDSBs, left panel), or induces fork reversal, providing a double-ended DNA end. As shown at deDSBs, RNA polymerase may engage at those DNA ends to generate focal RNAs at 
replication stress sites (represented as a yellow cloud) with potential role in fostering DDR signaling, in promoting RNA-templated fork repair and in modulating NHEJ activity during replication. NHEJ factors identified in large-scale proteomic studies as putative RNA Binding Proteins (RBPs) are underscored. Blue and orange lines indicate DNA and RNA, respectively.

\section{Text box: Focal RNAs at deDSBs: synthesis and functions}

At deDSBs, MRN allows RNAPII recruitment for de novo synthesis of RNAs called dilncRNAs (damage-induced long non coding RNAs), at both DNA ends [34, 36, 37 44]. At transcribed regions, dilncRNAs are complementary to pre-existing transcripts, to form double-stranded RNAs, that are further processed by RNase III enzyme such as human DICER to generate DDRNAs (DNA Damage Response RNAs) of around 21 ribonucleotides in size [35, 37, 42]. Also, when targeting DSBs within repeated sequences, multiple DSB-induced transcription events would generate complementary dilncRNAs to form dsRNAs. At a non-transcribed and unique region, bi-directional transcription from both sides of DSBs would generate complementary dilncRNAs to form dsRNAs. DDRNAs are involved in multiple ways within the repair, from constituting sequence-specific targets for DDR factors recruitment to fine-tuning the repair process itself. For instance, DDRNAs, together with dilncRNAs, are reported to foster DNA Damage Response (DDR) signaling by promoting phase separation [38]. Focal RNAs at deDSBs may form RNA:DNA hybrids acting positively by favoring repair factors recruitment or when persisting, creating a need for the recruitment of RNA:DNA hybrid dismantling activities [39-41, 43, 49, 50]. 


\section{Glossary box}

Homologous Recombination (HR) is a DSB repair pathway, initiated by a tightly orchestrated nucleolytic degradation of DSB ends to expose single-stranded DNA (ssDNA) on which the ssDNA-binding protein RPA (for Replication Protein A) is loaded. With the help of HR mediators, such as human BRCA2 and yeast Rad52, the recombinase RAD51 is then loaded onto SsDNA to form a well-structured nucleoprotein filament, responsible for search homology

Non-Homologous End-Joining (NHEJ) pathway acts at deDSBs by rejoining the two DNA extremities. Classical NHEJ (CNHEJ) is initiated by the binding of the heterodimer Ku70-Ku80 (also known as XRCC5 and XRCC6) to each double-stranded DNA ends. Ku binding further engages the DNA-dependent protein kinase catalytic subunit (DNA$\mathrm{PKCs}$ ), forming the DNA-PK holocomplex.

Alternative End-Joining (Alt-EJ) is an error-prone DSB repair mechanism alternative to canonical Non-Homologous End-Joining acting when a limited end-resection is engaged at deDSBs and that exploits microhomology to anneal and ligate DNA ends.

Replication stress (RS) corresponds to any event that distort the DNA replication program. A main feature of RS is the alteration of replication fork progression, either by a slow-down or an acceleration, resulting in stalled, collapsed or broken forks.

R-loop is a co-transcriptional three-stranded nucleic acid structure in which the nascent RNA anneals with its template DNA strand to form an RNA:DNA hybrid and a displaced single-stranded DNA.

DNA Damage Response (DDR) refers to as the cellular response to DNA damaging agents, including the molecular pathways to sense, signal and repair DNA lesions

BRCA1 and BRCA2 stand for Breast Cancer 1 and 2, respectively. They are genes for which the mutation or silencing is associated to human breast or ovarian cancer susceptibility. They encode for proteins involved in the DNA damage signalling and repair.

RNA Binding Protein (RBP) is a class of protein that directly binds RNA

Damage-induced long non-coding RNAs (dilncRNAs) are actively transcribed at deDSBs by RNAPII and contribute to form long double-stranded RNAs, further converted into shorter DNA Damage Response RNAs (DDRNAs), of around 21 ribonucleotides in size, by RNAse III enzymes.

RNA:DNA hybrids are nucleic acid structure in which an RNA is annealed to a complementary DNA template. Depending on their origin, the RNA moiety can be fully embedded in DNA or has unligated 3' and or 5' ends.

Okazaki fragments (OFs) are discontinuous newly replicated DNA stretches formed on the lagging strand. 
Quelling pathway, is a Neurospora crassa RNAi-related mechanism that posttranscriptionally silence repetitive DNA and transposons, a type of mobile DNA element.

G-quadruplexes are secondary structures that form in nucleic acids when the sequence is rich in guanine.

\section{Acknowledgments}

This work was supported by funding from Institut Curie, CNRS and INSERM as well as by the ANR grant REDEFINE (ANR-19-CE12-0016-03) to SL and the fondation LIGUE contre le cancer "Equipe Labellisée 2020 (EL2020LNCC/Sal) to SL. AC was funded by a French governmental fellowship and a $4^{\text {th }}$-year PhD grant from Fondation ARC. 


\section{References}

1 Ciccia, A. and Elledge, S.J. (2010) The DNA Damage Response: Making It Safe to Play with Knives. Mol. Cell 40, 179-204

2 Ait Saada, A. et al. (2018) Preserving replication fork integrity and competence via the homologous recombination pathway. DNA Repair (Amst). 71, 135-147

3 Zhao, B. et al. (2020) The molecular basis and disease relevance of nonhomologous DNA end joining. Nat. Rev. Mol. Cell Biol. 21, 765-781

4 Marini, F. et al. (2019) Regulation of DNA Double Strand Breaks Processing: Focus on Barriers. Frontiers in Molecular Biosciences. 6. 55

5 Zeman, M.K. and Cimprich, K.A. (2014) Causes and consequences of replication stress. Nat. Cell Biol. 16, 2-9

6 Saintigny, Y. et al. (2001) Characterization of homologous recombination induced by replication inhibition in mammalian cells. EMBO J. 20, 3861-3870

7 Miyoshi, T. et al. (2009) Fission yeast Ku protein is required for recovery from DNA replication stress. Genes to Cells 14, 1091-1103

8 Ribeyre, C. et al. (2016) Nascent DNA Proteomics Reveals a Chromatin Remodeler Required for Topoisomerase I Loading at Replication Forks. Cell Rep. 15, 300-309

9 Mukherjee, C. et al. (2019) RIF1 promotes replication fork protection and efficient restart to maintain genome stability. Nat. Commun. 10, 3287

10 Dungrawala, H. et al. (2015) The Replication Checkpoint Prevents Two Types of Fork Collapse without Regulating Replisome Stability. Mol. Cell. 59, 9981010

11 Kais, Z. et al. (2016) FANCD2 Maintains Fork Stability in BRCA1/2-Deficient Tumors and Promotes Alternative End-Joining DNA Repair. Cell Rep. 15, 2488-2499

12 Wang, Z. et al. (2019) DNA polymerase (POLQ) is important for repair of DNA double-strand breaks caused by fork collapse. J. Biol. Chem. 294, 3909-3919

13 Chappidi, N. et al. (2020) Fork Cleavage-Religation Cycle and Active Transcription Mediate Replication Restart after Fork Stalling at Cotranscriptional R-Loops. Mol. Cell. 77, 528-541

14 Joshi, R.R. et al. (2019) DNA Ligase IV Prevents Replication Fork Stalling and Promotes Cellular Proliferation in Triple Negative Breast Cancer. J. Nucleic Acids. 9170341

15 Ensminger, M. and Löbrich, M. (2020) One end to rule them all: Nonhomologous end-joining and homologous recombination at DNA double-strand breaks. The British journal of radiology. 93, 20191054

16 Whelan, D.R. et al. (2020) Super-resolution visualization of distinct stalled and broken replication fork structures. PLoS Genet. 16, e1009256 
17 Jones, C.E. and Forsburg, S.L. (2021) Monitoring Schizosaccharomyces pombe genome stress by visualizing end-binding protein Ku . Biol. Open. 10, bio054346

18 Foster, S.S. et al. (2011) Functional Interplay of the Mre11 Nuclease and Ku in the Response to Replication-Associated DNA Damage. Mol. Cell. Biol. 31, 4379-4389

19 Teixeira-Silva, A. et al. (2017) The end-joining factor Ku acts in the endresection of double strand break-free arrested replication forks. Nat. Commun. 8,1982

20 Mimitou, E.P. and Symington, L.S. (2010) Ku prevents Exo1 and Sgs1dependent resection of DNA ends in the absence of a functional MRX complex or Sae2. EMBO J. 29, 3358-3369

21 Balestrini, A. et al. (2013) The Ku Heterodimer and the Metabolism of SingleEnded DNA Double-Strand Breaks. Cell Rep. 3, 2033-2045

22 Chanut, P. et al. (2016) Coordinated nuclease activities counteract Ku at single-ended DNA double-strand breaks. Nat. Commun. 7, 12889

23 Pasero, P. and Vindigni, A. (2017) Nucleases Acting at Stalled Forks: How to Reboot the Replication Program with a Few Shortcuts. Annual Review of Genetics. 51, 477-499

24 Berti, M. et al. (2020) The plasticity of DNA replication forks in response to clinically relevant genotoxic stress. Nature Reviews Molecular Cell Biology. 21, 633-651

25 Sánchez, A. and Russell, P. (2015) Ku Stabilizes replication forks in the absence of Brc1. PLoS One. 10, e0126598

26 Liu, W. et al. (2020) Two replication fork remodeling pathways generate nuclease substrates for distinct fork protection factors. Sci. Adv. 6, eabc3598

27 Garzón, J. et al. (2019) Human RIF1-Protein Phosphatase 1 Prevents Degradation and Breakage of Nascent DNA on Replication Stalling. Cell Rep. $27,2558-2566$

28 Chen, B.R. et al. (2019) XLF and H2AX function in series to promote replication fork stability. J. Cell Biol. 218, 2113-2123

29 Balmus, G. et al. (2019) ATM orchestrates the DNA-damage response to counter toxic non-homologous end-joining at broken replication forks. Nat. Commun. 10, 87

30 Britton, S. et al. (2020) ATM antagonizes NHEJ proteins assembly and DNAends synapsis at single-ended DNA double strand breaks. Nucleic Acids Res. 48, 9710-9723

31 Willis, N.A. et al. (2017) Mechanism of tandem duplication formation in BRCA1mutant cells. Nature. 551, 590-595

32 Arnoult, N. et al. (2017) Regulation of DNA repair pathway choice in S and G2 phases by the NHEJ inhibitor CYREN. Nature. 549, 548-552 
33 Zong, D. et al. (2020) RNA: a double-edged sword in genome maintenance. Nature Reviews Genetics. 21, 651-670

34 Sharma, S. et al. (2021) MRE11-RAD50-NBS1 Complex Is Sufficient to Promote Transcription by RNA Polymerase II at Double-Strand Breaks by Melting DNA Ends. Cell Rep. 34, 108565

35 Francia, S. et al. (2012) Site-specific DICER and DROSHA RNA products control the DNA-damage response. Nature. 488, 231-235

36 Michelini, F. et al. (2017) Damage-induced IncRNAs control the DNA damage response through interaction with DDRNAs at individual double-strand breaks. Nat. Cell Biol. 19, 1400-1411

37 Bonath, F. et al. (2018) Next-generation sequencing reveals two populations of damage-induced small RNAs at endogenous DNA double-strand breaks. Nucleic Acids Res. 46, 11869-11882

38 Pessina, F. et al. (2019) Functional transcription promoters at DNA doublestrand breaks mediate RNA-driven phase separation of damage-response factors. Nat. Cell Biol. 21, 1286-1299

39 Ohle, C. et al. (2016) Transient RNA-DNA Hybrids Are Required for Efficient Double-Strand Break Repair. Cell. 167, 1001-1013

40 Cohen, S. et al. (2018) Senataxin resolves RNA:DNA hybrids forming at DNA double-strand breaks to prevent translocations. Nat. Commun. 9, 533

41 Yasuhara, T. et al. (2018) Human Rad52 Promotes XPG-Mediated R-loop Processing to Initiate Transcription-Associated Homologous Recombination Repair. Cell. 175, 558-570

42 Lu, W.T. et al. (2018) Drosha drives the formation of DNA:RNA hybrids around DNA break sites to facilitate DNA repair. Nat. Commun. 9, 532

43 D'Alessandro, G. et al. (2018) BRCA2 controls DNA:RNA hybrid level at DSBs by mediating RNase $\mathrm{H} 2$ recruitment. Nat. Commun. 9, 5376

44 Liu, S. et al. (2021) RNA polymerase III is required for the repair of DNA double-strand breaks by homologous recombination. Cell. 184, 1314-1329

45 Rawal, C.C. et al. (2020) Senataxin Ortholog Sen1 Limits DNA:RNA Hybrid Accumulation at DNA Double-Strand Breaks to Control End Resection and Repair Fidelity. Cell Rep. 31, 107603

46 Domingo-Prim, J. et al. (2020) RNA at DNA Double-Strand Breaks: The Challenge of Dealing with DNA:RNA Hybrids. BioEssays. 42, 1900225

47 Daley, J.M. et al. (2020) Specificity of end resection pathways for double-strand break regions containing ribonucleotides and base lesions. Nat. Commun. 11, 3088

48 Domingo-Prim, J. et al. (2019) EXOSC10 is required for RPA assembly and controlled DNA end resection at DNA double-strand breaks. Nat. Commun. 10, 2135

49 Sessa, G. et al. (2021) BRCA2 promotes DNA-RNA hybrid resolution by DDX5 
helicase at DNA breaks to facilitate their repair. EMBO J. 40, e106018

50 Li, L. et al. (2016) DEAD Box 1 Facilitates Removal of RNA and Homologous Recombination at DNA Double-Strand Breaks. Mol. Cell. Biol. 36, 2794-2810

51 Ghodgaonkar, M.M. et al. (2013) Ribonucleotides misincorporated into DNA act as strand-discrimination signals in eukaryotic mismatch repair. Mol. Cell. 50, 323-332

52 Burgers, P.M.J. and Kunkel, T.A. (2017) Eukaryotic DNA replication fork. , Annual Review of Biochemistry. 86, 417-438

53 Schmid, J.A. et al. (2018) Histone Ubiquitination by the DNA Damage Response Is Required for Efficient DNA Replication in Unperturbed S Phase. Mol. Cell. 71, 897-910

54 Yang, Q. et al. (2015) Mechanism of siRNA production from repetitive DNA. Genes Dev. 29, 526-537

55 Zhang, Z. et al. (2013) Homologous recombination as a mechanism to recognize repetitive DNA sequences in an RNAi pathway. Genes Dev. 27, $145-150$

56 Crossley, M.P. et al. (2019) R-Loops as Cellular Regulators and Genomic Threats. Mol. Cell. 73, 398-411

57 García-Muse, T. and Aguilera, A. (2019) R Loops: From Physiological to Pathological Roles. Cell. 179, 604-618

58 Stewart-Morgan, K.R. et al. (2019) Transcription Restart Establishes Chromatin Accessibility after DNA Replication. Mol. Cell. 75, 284-297

59 Appanah, R. et al. (2020) Sen1 Is Recruited to Replication Forks via Ctf4 and Mrc1 and Promotes Genome Stability. Cell Rep. 30, 2094-2105.e9

60 Kim, S. et al. (2020) ATAD5 restricts R-loop formation through PCNA unloading and RNA helicase maintenance at the replication fork. Nucleic Acids Res. 48, 7218-7238

61 Alzu, A. et al. (2012) Senataxin associates with replication forks to protect fork integrity across RNA-polymerase-II-transcribed genes. Cell. 151, 835-846

62 Conrad, T. et al. (2016) Serial interactome capture of the human cell nucleus. Nat. Commun. 7, 11212

63 Baltz, A.G. et al. (2012) The mRNA-Bound Proteome and Its Global Occupancy Profile on Protein-Coding Transcripts. Mol. Cell. 46, 674-690

64 Beckmann, B.M. et al. (2015) The RNA-binding proteomes from yeast to man harbour conserved enigmRBPs. Nat. Commun. 6, 10127

65 Huang, R. et al. (2018) Transcriptome-wide discovery of coding and noncoding RNA-binding proteins. Proc. Natl. Acad. Sci. U. S. A. 115, 3879-3887

66 Queiroz, R.M.L. et al. (2019) Comprehensive identification of RNA-protein interactions in any organism using orthogonal organic phase separation (OOPS). Nat. Biotechnol. 37, 169-178 
67 Trendel, J. et al. (2019) The Human RNA-Binding Proteome and Its Dynamics during Translational Arrest. Cell. 176, 391-403

68 Castello, A. et al. (2016) Comprehensive Identification of RNA-Binding Domains in Human Cells. Mol. Cell. 63, 696-710

69 Bhatia, V. et al. (2018) Formation of 53BP1 foci and ATM activation under oxidative stress is facilitated by RNA:DNA hybrids and loss of ATM-53BP1 expression promotes photoreceptor cell survival in mice. F1000 Research. 7, 1233

70 Pryde, F. et al. (2005) 53BP1 exchanges slowly at the sites of DNA damage and appears to require RNA for its association with chromatin. J. Cell Sci. 118, 2043-2055

71 Boisvert, F.M. et al. (2005) The GAR motif of 53BP1 is arginine methylated by PRMT1 and is necessary for 53BP1 DNA binding activity. Cell Cycle. 4, 18341841

72 Yu, Z. et al. (2012) The MRE11 GAR motif regulates DNA double-strand break processing and ATR activation. Cell Res. 22, 305-320

73 Thandapani, P. et al. (2013) Defining the RGG/RG Motif. Molecular Cell. 50, 613-623

74 Drané, P. et al. (2017) TIRR regulates 53BP1 by masking its histone methyllysine binding function. Nature. 543, 211-216

75 Parnandi, N. et al. (2021) TIRR inhibits the 53BP1-p53 complex to alter cellfate programs. Mol. Cell. 81, 1-13

76 Betts, J.A. et al. (2017) Long Noncoding RNAs CUPID1 and CUPID2 Mediate Breast Cancer Risk at 11q13 by Modulating the Response to DNA Damage. Am. J. Hum. Genet. 101, 255-266

77 Dalby, A.B. et al. (2013) RNA recognition by the DNA end-binding Ku heterodimer. RNA 19, 841-851

78 Ting, N.S.Y. et al. (2005) Human Ku70/80 interacts directly with hTR, the RNA component of human telomerase. Nucleic Acids Res. 33, 2090-2098

79 Thapar, R. et al. (2020) Mechanism of efficient double-strand break repair by a long non-coding RNA. Nucleic Acids Res. 48, 10953-10972

80 Lamaa, A. et al. (2016) A novel cytoprotective function for the DNA repair protein $\mathrm{Ku}$ in regulating p53 mRNA translation and function. EMBO Rep. 17, 508-518

81 Adelmant, G. et al. (2012) DNA ends alter the molecular composition and localization of ku multicomponent complexes. Mol. Cell. Proteomics. 11, 411421

82 Zhang, Y. et al. (2016) Long noncoding RNA LINP1 regulates repair of DNA double-strand breaks in triple-negative breast cancer. Nat. Struct. Mol. Biol. 23, $522-530$

83 Shao, Z. et al. (2020) DNA-PKcs has KU-dependent function in rRNA 
processing and haematopoiesis. Nature 579, 291-296

84 Ting, N.S.Y. et al. (2009) The human telomerase RNA component, hTR, activates the DNA-dependent protein kinase to phosphorylate heterogeneous nuclear ribonucleoprotein A1. Nucleic Acids Res. 37, 6105-6115

85 Wang, D. et al. (2020) LRIK interacts with the Ku70-Ku80 heterodimer enhancing the efficiency of NHEJ repair. Cell Death Differ. 27, 3337-3353

86 Meers, C. et al. (2016) DNA repair by RNA: Templated, or not templated, that is the question. DNA Repair. 44, 17-21

87 Aymard, F. et al. (2014) Transcriptionally active chromatin recruits homologous recombination at DNA double-strand breaks. Nat. Struct. Mol. Biol. 21, 366374

88 Dang, T.T. and Morales, J.C. (2020) Xrn2 links RNA: Dna hybrid resolution to double strand break repair pathway choice. Cancers (Basel). 12, 1-15

89 Morales, J.C. et al. (2016) XRN2 Links Transcription Termination to DNA Damage and Replication Stress. PLoS Genet. 12, e1006107

90 Chakraborty, A. et al. (2016) Classical non-homologous end-joining pathway utilizes nascent RNA for error-free double-strand break repair of transcribed genes. Nat. Commun. 7, 13049

91 Wang, I.X. et al. (2018) Human proteins that interact with RNA/DNA hybrids. Genome Res. 28, 1405-1414

92 Puget, N. et al. (2019) Non-canonical DNA/RNA structures during Transcription-Coupled Double-Strand Break Repair: Roadblocks or Bona fide repair intermediates? DNA Repair. 81, 102661

93 Abakir, A. et al. (2020) N 6-methyladenosine regulates the stability of RNA:DNA hybrids in human cells. Nature Genetics. 52, 48-55

94 Pryor, J.M. et al. (2018) Ribonucleotide incorporation enables repair of chromosome breaks by nonhomologous end joining. Science. 361, 1126-1129

95 Frank-Vaillant, M. and Marcand, S. (2002) Transient stability of DNA ends allows nonhomologous end joining to precede homologous recombination. Mol. Cell. 10, 1189-1199

96 Sun, B. et al. (2018) Helicase promotes replication re-initiation from an RNA transcript. Nat. Commun. 9, 1-9

97 Mazina, O.M. et al. (2020) Replication protein A binds RNA and promotes Rloop formation. J. Biol. Chem. 295, 14203-14213

98 Gylling, H.M. et al. (2020) Repeat RNAs associate with replication forks and post-replicative DNA. RNA 26, 1104-1117 


\section{Highlights}

- Non-Homologous End-Joining (NHEJ), a double strand break (DSB) repair pathway, is active during DNA replication, as several NHEJ factors are involved in safeguarding the integrity of newly replicated strands, in repairing replication-induced DSBs and in restarting replication forks.

- RNAs at DSBs are instrumental in fueling the DNA Damage Response (DDR) and guiding-modulating the fidelity of DNA repair by NHEJ.

- RNAs are modulators of NHEJ activity acting in trans as scaffoldsers, or in cis to promote RNA-templated DSB repair, thereby regulating the repair pathway choice.

- Ribonucleotides co-exist in the DNA template during unstressed and stressed DNA replication and may influence NHEJ activity at halted replication forks. 


\section{Outstanding questions}

- Are RNAs transcribed at replication stress sites, as described at deDSBs, and what are the molecular mechanisms involved in such focal RNAs synthesis?. How is transcription coordinated with the processing of halted replication forks? To what extent such crosstalk is instrumental in maintaining replication fork stability and in sustaining replication dynamics upon replication stress? Understanding these mechanisms could open exciting opportunities to improve anti-cancer therapy.

- What is the repertoire of replication stress-induced ncRNAs? How their biogenesis is related to replication fork passage and fork stalling? Are there ncRNAs acting in cis or in trans to modulate NHEJ activity and guide replication fork-repair pathway choice?

- Are DNA-embedded ribonucleotides, associated to the process of DNA replication, instrumental to replication fork-repair? How such ribonucleotides influence the binding of NHEJ factors to various fork-substrates, including, seDSBs, deDSBs and dsDNA end from reversed fork? How are DNA-embedded ribonucleotides at halted forks used to modulate NHEJ?

- Are NHEJ and DDR factors directly interacting with RNAs? Is direct RNA binding important for their DNA repair function and DNA damage signaling functions? Do they contain non canonical RNA binding domains? Do they contain multiple RNA binding domains each involved in specific functions for deDSBs repair versus replication forkrepair?

- The role of NHEJ in resolving replication stress is exacerbated in cancer cells, contributing to their survival to replication-blocking agents. ncRNAs play critical roles during tumorigenesis by functioning as scaffolds to regulate protein-protein or proteinnucleic acids interactions. Can NHEJ-modulatory ncRNAs be targeted to synergize with actual DNA-damaging strategies used for cancer therapy? 


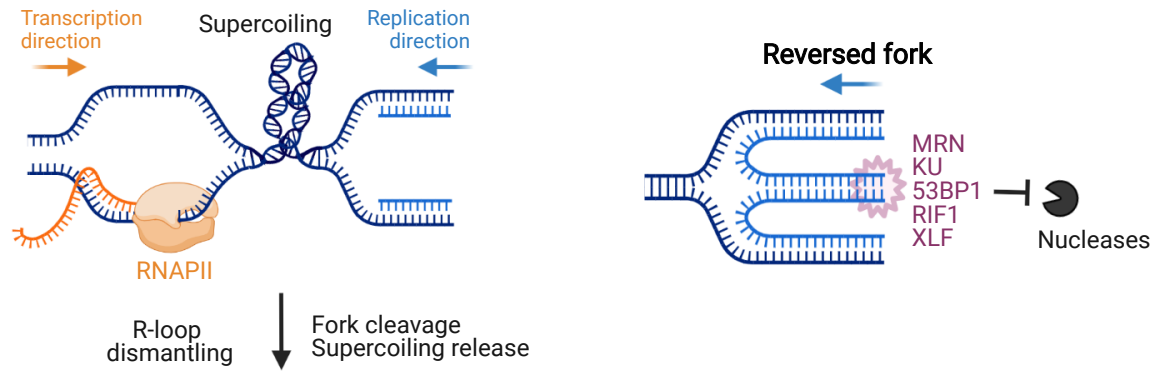

\section{C}
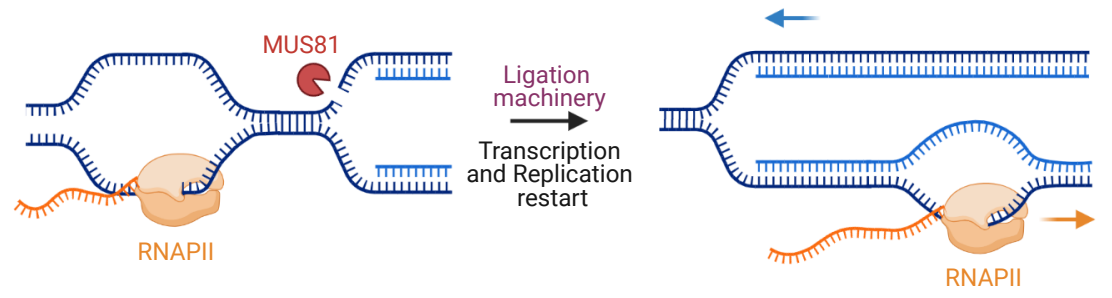

B

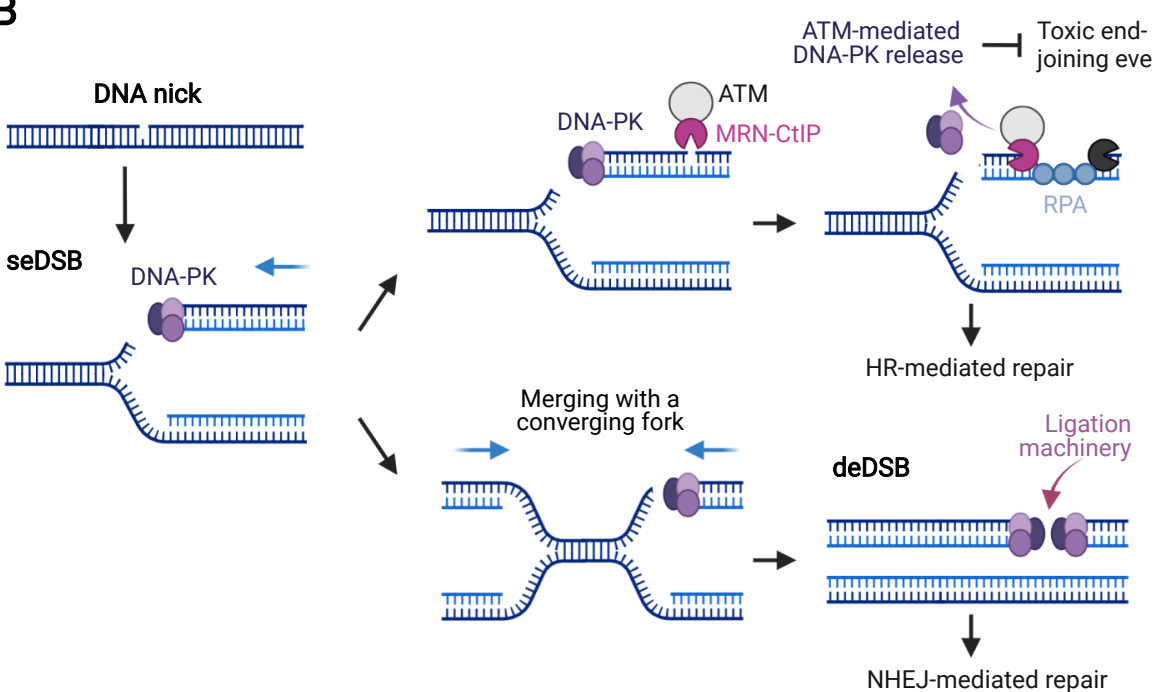

NHEJ-mediated repair 
A

ncRNAs at DSBs foster 53BP1-mediated DDR

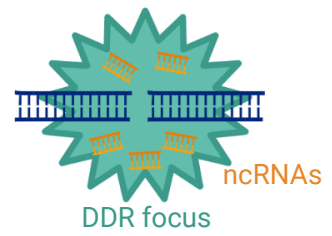

B RNA:DNA hybrids as modulators of NHEJ activity

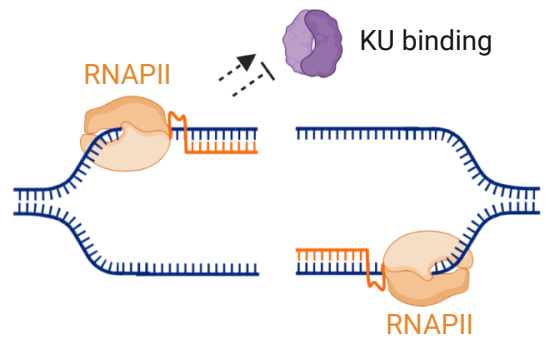

C RNA-templated DSB repair

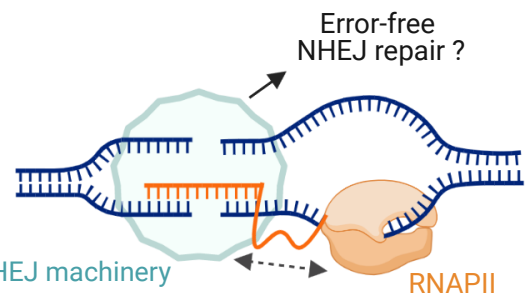

D IncRNAs as regulators of 53BP1 activity

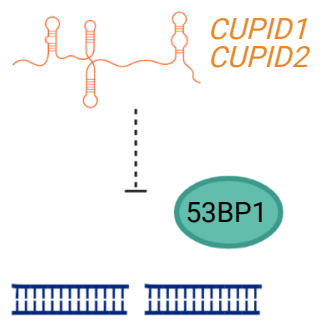

E IncRNAs as scaffolds of DNA-PK LINP1, TLC1

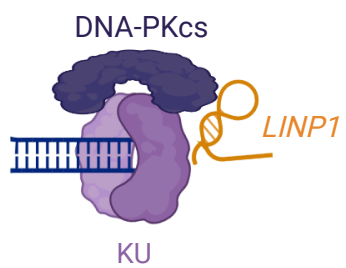




\section{Key figsure}

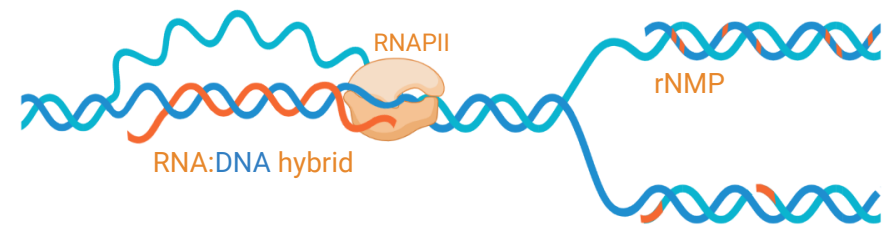

B RNAs during replication stress

\section{RNA primer}

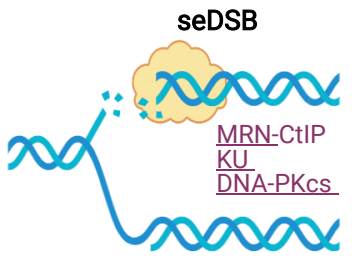

Merging with a converging fork
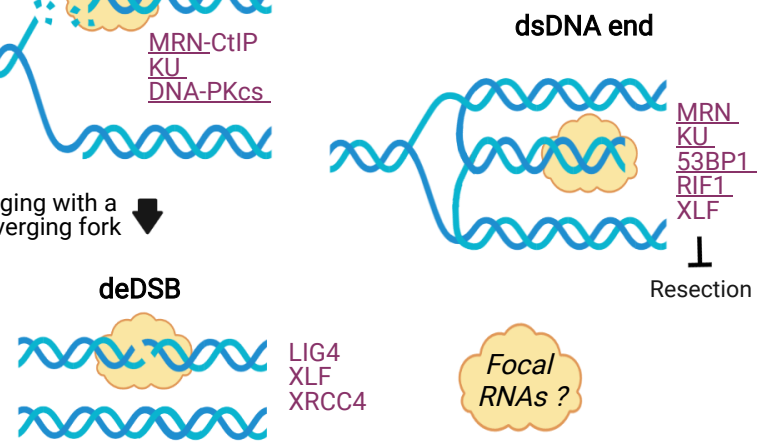

RNA-templated fork repair?

Impact on NHEJ activity/recruitment ?

Role of RNA Binding Proteins? 


\section{text box figure}
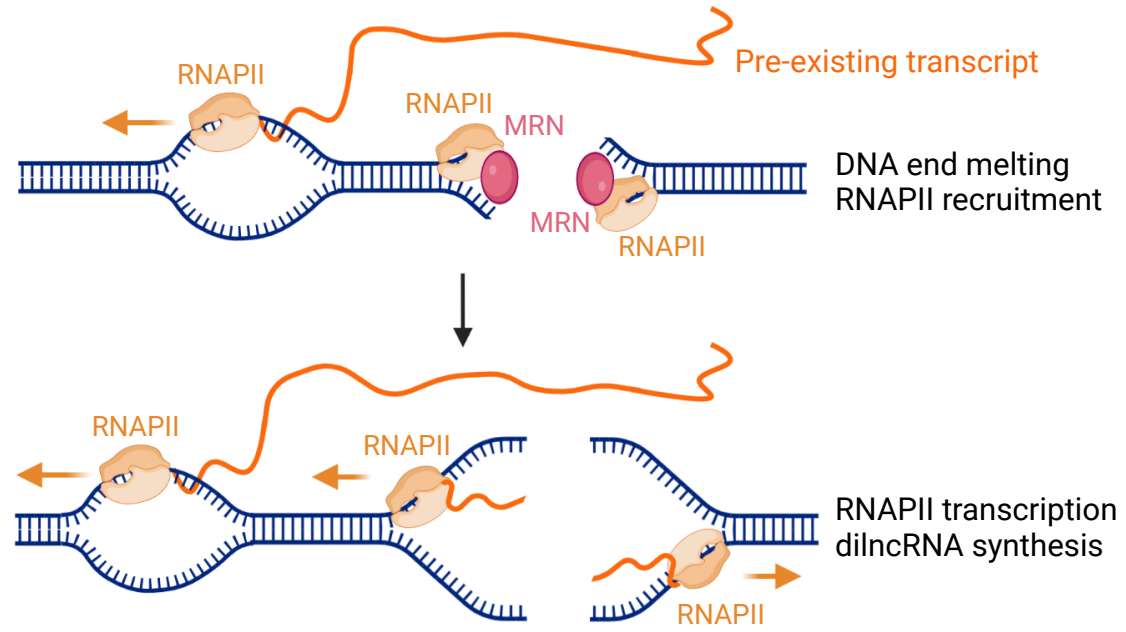

Damage-induced transcript

$\downarrow$

IIIIIIII DDRNA precursors

$\downarrow$ RNase III activities

DDR signaling Phase-separation

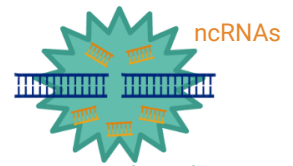

53BP1-mediated DDR
Iय)

IIII

IIII DDRNAs
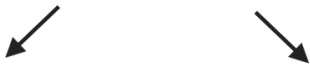

RNA:DNA hybrid formation

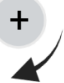

Repair factor recruitment
Require dismantling activities 